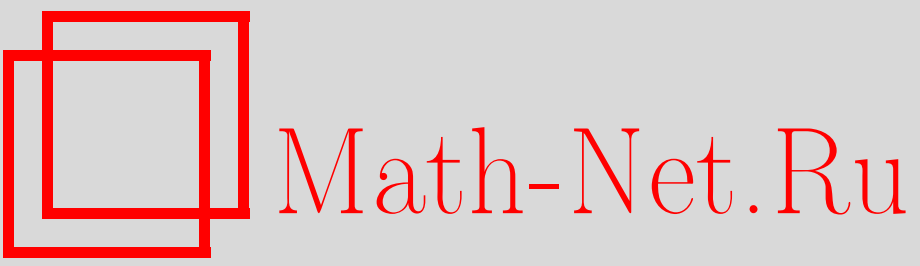

3. Муайн, Характеризация многомерных евклидовых состояний Ландау с помощью преобразований когерентных состояний, ТМФ, 2004, том 140, номер 3, 492499

DOI: https://doi.org/10.4213/tmf104

Использование Общероссийского математического портала Math-Net.Ru подразумевает, что вы прочитали и согласны с пользовательским соглашением http://www . mathnet.ru/rus/agreement

Параметры загрузки:

IP: 54.162 .127 .20

26 апреля 2023 г., 13:16:21 


\title{
ХАРАКТЕРИЗАЦИЯ МНОГОМЕРНЫХ ЕВКЛИДОВЫХ СОСТОЯНИЙ ЛАНДАУ С ПОМОШЬЮ ПРЕОБРАЗОВАНИЙ КОГЕРЕНТНЫХ СОСТОЯНИЙ
}

\begin{abstract}
Рассматривается семейство многомерных обобщенных когерентных состояний, получаемых посредством представления Фока-Баргмана группы Гейзенберга. Доказано, что области значений соответствующих преобразований когерентных состояний совпадают с пространствами связанных состояний системы, являющейся четномерным аналогом заряженной частицы в однородном магнитном поле. Это дает новое описание евклидовых состояний Ландау.
\end{abstract}

Ключевые слова: группа Гейзенберга, представление Фока-Баргмана, преобразования когерентных состояний, евклидовы состояния Ландау.

\section{1. ВВЕДЕНИЕ}

В последние несколько лет когерентные состояния (KC) привлекли к себе большое внимание. КС являются полезным инструментом в теории поля, и существует много различных определений $\mathrm{KC} \mathrm{(см.} \mathrm{[1]} \mathrm{и} \mathrm{приведенные} \mathrm{там} \mathrm{ссылки).}$

$\mathrm{KC}$ для заряженной частицы, движущейся в постоянном однородном магнитном поле, перпендикулярном евклидовой $x y$-плоскости, изучались в работах [2]-[4] и другими авторами. В этой задаче связанные с нижним уровнем Ландау КС были рассмотрены в [5], а для всех уровней Ландау КС были построены в [6].

В настоящей работе рассматривается четномерный аналог заряженной частицы в постоянном однородном магнитном поле. Для каждого уровня Ландау строится семейство обобщенных когерентных состояний (OKC). При построении используется метод, в основе которого лежат квадратично интегрируемые на однородном пространстве представления основной группы. Конкретно нас интересует представление Фока-Баргмана группы Гейзенберга $\mathbb{H}_{n}=\mathbb{C}^{n} \times \mathbb{R}$, реализованное на пространстве Фока $\mathcal{F}_{2}\left(\mathbb{C}^{n}\right)$ целых гауссовых квадратично интегрируемых функций. Установлено, что образы пространства Фока при преобразованиях КС, ассоциированных с построенными ОКС, совпадают с пространствами связанных состояний гамильтониана Ландау в $\mathbb{C}^{n}$.

* Department of Mathematics, Faculty of Sciences and Technics (M'Ghila), Cadi Ayyad University, Béni Mellal, Morocco. E-mail: mouayn@math.net 
Настояшая работа организована следуюшим образом. В разделе 2 мы представляем гамильтониан Ландау, действуюший на гильбертовом пространстве комплекснозначных гауссовых квадратично интегрируемых функций. В разделе 3 дано краткое описание пространств состояний Ландау. В разделе 4 мы даем обзор формализма построения KC с помощью представлений группы, которые являются квадратично интегрируемыми на факторпространстве группы по модулю подгруппы. Этот формализм применяется в разделе 5 к представлению Фока-Баргмана. В разделе 6 устанавливается теорема характеризации для состояний Ландау. В разделе 7 приведены некоторые заключительные замечания.

\section{2. ГАМИЛЬТОНИАН ЛАНДАУ В $\mathbb{C}^{n}$}

В подходящей системе единиц с точностью до аддитивной константы гамильтониан Ландау заряженной частицы в постоянном однородном магнитном поле в пространстве $\mathbb{R}^{2 n}$ имеет вид

$$
H_{B}=-\frac{1}{4} \sum_{j=1}^{n}\left(\left(\partial_{x_{j}}+i B y_{j}\right)^{2}+\left(\partial_{y_{j}}-i B x_{j}\right)^{2}\right)-\frac{n}{2}
$$

и действует на пространстве $L^{2}\left(\mathbb{R}^{2 n}, d \mu\right), d \mu$-мера Лебега на $\mathbb{R}^{2 n}$. Для того чтобы использовать комплексную структуру, отождествим евклидово пространство $\mathbb{R}^{2 n}$ с пространством $\mathbb{C}^{n}$ обычным способом. Для $\xi, \zeta \in \mathbb{C}^{n}, \xi=\left(\xi_{1}, \ldots, \xi_{n}\right), \xi_{j} \in \mathbb{C}$, пишем $\xi \cdot \zeta=\xi_{1} \zeta_{1}^{*}+\cdots+\xi_{n} \zeta_{n}^{*}, \zeta^{*}=\left(\zeta_{1}^{*}, \ldots, \zeta_{n}^{*}\right), \zeta_{j}^{*}-$ комплексно-сопряженное к $\zeta_{j} \in \mathbb{C}_{\text {и }}$ $|\xi|^{2}=\xi \cdot \xi$. Оператор, соответствующий $H_{B}(1)$, может быть представлен оператором

$$
\widetilde{H}_{B}:=e^{B|\xi|^{2} / 2} H_{B} e^{-B|\xi|^{2} / 2}
$$

В соответствии с (2) произвольный кет-вектор | $>$ представляется функцией

$$
\psi(\xi):=e^{B|\xi|^{2} / 2}\langle\xi \mid\rangle, \quad \psi \in L^{2}\left(\mathbb{C}^{n}, e^{-B|\xi|^{2}} d \mu\right)
$$

Явное выражение для оператора (2) имеет вид

$$
\widetilde{H}_{B}=-\sum_{j=1}^{n} \frac{\partial^{2}}{\partial \xi_{j} \partial \xi_{j}^{*}}+B \sum_{j=1}^{n} \xi_{j}^{*} \frac{\partial}{\partial \xi_{j}^{*}}
$$

Далее мы полагаем в (3) $B=1$ и рассматриваем гамильтониан Ландау $\widetilde{H}:=\widetilde{H}_{1}$, действуюший на гильбертовом пространстве $\mathcal{H}:=L^{2}\left(\mathbb{C}^{n}, e^{-|\xi|^{2}} d \mu\right)$, которое снабжено эрмитовым скалярным произведением

$$
\langle\psi, \varphi\rangle_{\mathcal{H}}=\int_{\mathbb{C}^{n}} d \mu(\xi) e^{-|\xi|^{2}} \psi(\xi) \varphi(\xi)^{*}
$$




\section{3. ПРОСТРАНСТВА СОСТОЯНИЙ ЛАНДАУ}

Представим краткое описание пространств состояний Ландау, определенных как пространства связанных состояний гамильтониана $\widetilde{H}$. Прежде всего введем некоторые обозначения. Для $p, q \in \mathbb{Z}_{+}$обозначим через $\mathcal{Y}_{p, q}$ пространство сужений на сферу $\mathbf{S}^{2 n-1}=$ $\left\{\omega \in \mathbb{C}^{n},|\omega|=1\right\}$ однородных (степени $p$ по переменной $\xi$ и степени $q$ по переменной $\xi^{*}$ ) евклидовых гармонических полиномов на пространстве $\mathbb{C}^{n}$. При $n=2,3, \ldots$ размерность пространства $\mathcal{Y}_{p, q}$ равна

$$
d(n, p, q)=\frac{(p+q-1)(p+n-2) !(q+n-2) !}{p ! q !(n-1) !(n-2) !},
$$

а для $n=1$ имеем $p q=0$, и в этом случае $d(1, p, q)=1$. Более подробную информацию относительно этих сферических гармоник можно найти в [7], [8].

Для $\lambda \in \mathbb{C}$ обозначим через $\mathcal{A}_{\lambda}^{2}\left(\mathbb{C}^{n}\right)$ пространство собственных состояний $|\psi\rangle$ гамильтониана $\widetilde{H}$ в пространстве $\mathcal{H}$ с собственным значением $\lambda$. Полное описание этого собственного пространства, приведенное в [9], можно суммировать следующим образом. Если $\lambda \neq 0,1,2, \ldots$, то $\mathcal{A}_{\lambda}^{2}\left(\mathbb{C}^{n}\right)$ - тривиальное пространство. Если $\lambda=m \in \mathbb{Z}_{+}$, то волновая функция состояния $|\psi\rangle$ есть функция

$$
\langle\xi \mid \psi\rangle=\sum_{\substack{p \geqslant 0 \\ 0 \leqslant q \leqslant m}} \sum_{1 \leqslant j \leqslant d(n, p, q)} \frac{(m-q) ! \Gamma(n+p+q)}{\Gamma(n+p+m)} L_{m-q}^{p+q+n-1}\left(r^{2}\right) r^{p+q} \gamma_{p, q}^{j} h_{p, q}^{j}(\omega),
$$

принадлежашая $C^{\infty}\left(\mathbb{C}^{n}\right)$, где $\xi=r \omega, \omega \in \mathbf{S}^{2 n-1}, \quad r>0, L_{k}^{\beta}(x)$ - полином Лагерра (см. [10]), $\left\{h_{p, q}^{j}\right\}_{1 \leqslant j \leqslant d(n, p, q)}$ - ортонормированный базис в $\mathcal{Y}_{p, q}$ и $\left(\gamma_{p, q}^{j}\right)=\gamma_{p, q} \in$ $\mathbb{C}^{d(n, p, q)}$ таковы, что

$$
\sum_{\substack{p \geqslant 0 \\ 0 \leqslant q \leqslant m}} \frac{(m-q) !(p+q+n-1) ! \Gamma(n+p+q)}{2 \Gamma(n+p+m)}\left|\gamma_{p, q}\right|^{2}<+\infty .
$$

\section{4. ТЕОРЕТИКО-ГРУППОВОЙ ФОРМАЛИЗМ}

Пусть $G$ - локально компактная группа, $U$ - замкнутая подгруппа, $X=G / U$ - соответствуюшее однородное пространство с мерой $\nu$, инвариантной относительно действия группы $G$. Пусть $\sigma: X \rightarrow G$ - борелевское сечение (в главном расслоении $G \rightarrow G / U$ ) и $T$ - непрерывное унитарное представление группы $G$ обратимыми изометричными операторами $T(g), g \in G$, в гильбертовом пространстве $\mathrm{H}$.

В соответствии с [11] будем говорить, что $\phi_{0} \in \mathrm{H}$ есть вакуумный вектор, если для Bcex $u \in U$

$$
T(u)\left[\phi_{0}\right]=\chi(u) \phi_{0} .
$$

Отображение $u \rightarrow \chi(u)$ определяет характер подгруппы $U$. Будем говорить, что множество

$$
\phi_{x}:=T(\sigma(x))\left[\phi_{0}\right], \quad x \in X,
$$


образует семейство КС, если

$$
\int_{X} d \nu(x)\left\langle\phi_{0}, \phi_{x}\right\rangle\left\langle\phi_{x}, \phi_{0}\right\rangle=\left\langle\phi_{0}, \phi_{0}\right\rangle .
$$

Преобразование $\mathrm{KC}$ может быть определено как отображение из пространства $\mathrm{H}$ в пространство ограниченных непрерьвных функций на $G$ с помошью коэффициентов представления

$$
\phi \rightarrow\left\langle\phi, T(g)\left[\phi_{0}\right]\right\rangle, \quad g \in G .
$$

Вследствие (4) такие функции обладают простыми трансформационными свойствами при движении вдоль орбит $g U$, т.е. $\left\langle\phi, T(g u)\left[\phi_{0}\right]\right\rangle=\chi(u)^{*}\left\langle\phi, T(g)\left[\phi_{0}\right]\right\rangle, u \in U$, они полностью определяются своими значениями, проиндексированными точками пространства $X$. Поэтому мы можем рассматривать редуцированные преобразования $\mathrm{KC}$ из гильбертова пространства Н в пространство $L^{2}(X, d \nu)$, определенные формулой [11]

$$
W: \phi \rightarrow W[\phi](x):=\left\langle\phi, \phi_{x}\right\rangle_{\mathcal{H}} .
$$

\section{5. ПРЕДСТАВЛЕНИЕ ФОКА-БАРГМАНА И КОГЕРЕНТНЫЕ СОСТОЯНИЯ}

Группа Гейзенберга $\mathbb{H}_{n}$ - это группа Ли, являюшаяся многообразием $\mathbb{C}^{n} \times \mathbb{R}$ с умножением

$$
(\xi, t)(\zeta, s)=\left(\xi+\zeta, t+s+\frac{1}{2} \operatorname{Im}(\zeta \cdot \xi)\right),
$$

где $\operatorname{Im}(\zeta \cdot \xi)=(\zeta \cdot \xi-\xi \cdot \zeta) /(2 i)$. Группа $\mathbb{H}_{n}$ является унимодулярной группой, мера Хаара которой совпадает с мерой Лебега на $\mathbb{C}^{n} \times \mathbb{R}$.

Сильно непрерывные унитарные неприводимые представления (УНП) группы $\mathbb{H}_{n}$ хорошо известны $([12]$, с. 37). Здесь мы сосредоточим внимание на обозначаемом через $\rho$ УНП группы $\mathbb{H}_{n}$, которое реализуется на пространстве Фока $\mathcal{F}_{2}\left(\mathbb{C}^{n}\right)$ целых функций $\varphi$ на $\mathbb{C}^{n}$ таких, что $\langle\varphi, \varphi\rangle_{\mathcal{H}}<+\infty$, следуюшим образом:

$$
\rho(\xi, t)[\varphi](z)=e^{i t} e^{z \cdot \xi^{*}-|\xi|^{2} / 2} \varphi\left(z-\xi^{*}\right),
$$

$\varphi \in \mathcal{F}_{2}\left(\mathbb{C}^{n}\right), z \in \mathbb{C}^{n}$, и называется представлением Фока-Баргмана группы $\mathbb{H}_{n}$. УНП $\rho$ квадратично интегрируемо в следующем смысле: если $\mathbb{R}$ - центр группы $\mathbb{H}_{n}$ и сечение $\sigma_{0}$ - сечение расслоения $\mathbb{H}_{n} \rightarrow \mathbb{H}_{n} / \mathbb{R}=\mathbb{C}^{n}$, определенное формулой $\sigma_{0}(\xi)=(\xi, 0)$ [13], то сушествует ненулевой вектор $\phi_{0} \in \mathcal{F}_{2}\left(\mathbb{C}^{n}\right)$ такой, что

$$
\int_{\mathbb{H}_{n} / \mathbb{R}}\left|\left\langle\rho\left(\sigma_{0}(\xi)\right)\left[\phi_{0}\right], \phi_{0}\right\rangle_{\mathcal{H}}\right|^{2} d \mu(\xi)<+\infty .
$$

Это условие может быть также выражено как сушествование некоторого самосопряженного положительного полуинвариантного оператора $K$ в пространстве $\mathcal{F}_{2}\left(\mathbb{C}^{n}\right)$ такого, что

$$
\int_{\mathbb{C}^{n}} d \mu(\xi)\left\langle\varphi_{1}, \rho(\xi, 0) \psi_{1}\right\rangle\left\langle\rho(\xi, 0) \psi_{2}, \varphi_{2}\right\rangle=\left\langle\varphi_{1}, \varphi_{2}\right\rangle\left\langle K^{1 / 2} \psi_{1}, K^{1 / 2} \psi_{2}\right\rangle
$$


для всех $\psi_{1}, \psi_{2} \in \operatorname{dom}\left(K^{1 / 2}\right)$ и всех $\varphi_{1}, \varphi_{2} \in \mathcal{F}_{2}\left(\mathbb{C}^{n}\right)$. Поскольку группа $\mathbb{H}_{n}$ унимодулярная, оператор $K$ здесь тождественный [14].

Теперь в соответствии с (5) построим множество ОKС

$$
|\xi, \alpha\rangle:=\rho\left(\sigma_{0}(\xi)\right)|\alpha\rangle,
$$

$\xi \in \mathbb{C}^{n}$, и $|\alpha\rangle$ - нормированное фоковское состояние с волновой функцией

$$
\langle z \mid \alpha\rangle:=\frac{1}{\sqrt{\pi^{n} \alpha !}} z^{\alpha},
$$

$\alpha \in\left(\mathbb{Z}_{+}\right)^{n},|\alpha|=\alpha_{1}+\cdots+\alpha_{n}=m$, которое представляет вакуумный вектор, удовлетворяюший (4). Волновые функции $\mathrm{OKC}|\xi, \alpha\rangle$, определенные в (10), даются тогда выражением

$$
\langle z \mid \xi, \alpha\rangle:=\frac{1}{\sqrt{\pi^{n} \alpha !}} e^{z \cdot \xi^{*}-|\xi|^{2} / 2}\left(z-\xi^{*}\right)^{\alpha}, \quad z \in \mathbb{C}^{n}
$$

Такое определение ОКС полностью оправдывается квадратичной интегрируемостью $\rho$ по модулю подгруппы $\mathbb{R}$. Действительно, из условия (9) вытекает условие (6), которое в нашем случае имеет вид

$$
\int_{\mathbb{C}^{n}} d \mu(\xi)\langle\varphi \mid \xi, \alpha\rangle\langle\varphi \mid \xi, \alpha\rangle^{*}=\langle\varphi, \varphi\rangle, \quad \varphi \in \mathcal{F}_{2}\left(\mathbb{C}^{n}\right) .
$$

\section{6. ХАРАКТЕРИЗАЦИЯ СОСТОЯНИЙ ЛАНДАУ}

Прежде всего заметим, что условие (11) означает, что редуцированное преобразование $\mathrm{KC} \mathcal{W}_{m}: \mathcal{F}_{2}\left(\mathbb{C}^{n}\right) \rightarrow L^{2}\left(\mathbb{C}^{n}, d \mu\right)$, определенное в соответствии с $(7)$ формулой

$$
\mathcal{W}_{m}[\varphi](\xi):=\int_{\mathbb{C}^{n}} d \mu(z) e^{-|z|^{2}} \varphi(z)^{*}\langle z \mid \xi, \alpha\rangle,
$$

есть изометрическое вложение. Однако для того чтобы охарактеризовать пространства состояний Ландау $\mathcal{A}_{m}^{2}\left(\mathbb{C}^{n}\right)$, которые включены в гильбертово пространство $\mathcal{H}$ квадратично интегрируемых с весом $e^{-|\xi|^{2}} d \mu$ функций, мы должны воспользоваться унитарным преобразованием $\widetilde{\mathcal{W}}:=Q \circ \mathcal{W}_{m}$, в котором $Q: L^{2}\left(\mathbb{C}^{n}, d \mu\right) \rightarrow \mathcal{H}$ есть унитарное отображение (преобразование основного состояния), определенное формулой $Q[\phi](\xi)=$ $e^{|\xi|^{2} / 2} \phi(\xi), \xi \in \mathbb{C}^{n}$

Теперь мы можем сформулировать следующую теорему характеризации.

Теорема. Для $m \in \mathbb{Z}_{+}$имеет место равенство

$$
\widetilde{\mathcal{W}}_{m}\left[\mathcal{F}_{2}\left(\mathbb{C}^{n}\right)\right]=\mathcal{A}_{m}^{2}\left(\mathbb{C}^{n}\right)
$$

Для того чтобы показать, что $\widetilde{\mathcal{W}}_{m}\left[\mathcal{F}_{2}\left(\mathbb{C}^{n}\right)\right] \subset \mathcal{A}_{m}^{2}\left(\mathbb{C}^{n}\right)$, нам необходимо вычислить действие гамильтониана Ландау $\widetilde{H}\left(H_{1}\right.$ в формуле $\left.(3)\right)$ на $\widetilde{\mathcal{W}}_{m}[\varphi](\xi)$ для произвольной функции $\varphi \in \mathcal{F}_{2}\left(\mathbb{C}^{n}\right), \xi \in \mathbb{C}^{n}$. Прямое вычисление показывает, что $\widetilde{H} \widetilde{\mathcal{W}}_{m}[\varphi](\xi)=$ $m \widetilde{\mathcal{W}}_{m}[\varphi](\xi)$. 
Обратно, пусть $\psi \in \mathcal{A}_{m}^{2}\left(\mathbb{C}^{n}\right)$. Рассмотрим функцию

$$
\varphi_{\psi}(z):=\pi^{-n} \sum_{|\alpha|=m} \int_{\mathbb{C}^{n}} d \mu(\zeta) e^{-|\zeta|^{2} / 2}(\psi(\zeta))^{*}\langle z \mid \zeta, \alpha\rangle .
$$

Покажем, что $\varphi_{\psi}$ удовлетворяет условию $\widetilde{\mathcal{W}}_{m}\left[\varphi_{\psi}\right]=\psi$ :

$$
\begin{aligned}
& \widetilde{\mathcal{W}}_{m}\left[\varphi_{\psi}\right](\xi)=\pi^{-n} e^{|\xi|^{2} / 2} \int_{\mathbb{C}^{n}} d \mu(z) e^{-|z|^{2}}\langle z \mid \xi, \alpha\rangle \times \\
& \times\left(\sum_{|\alpha|=m} \int_{\mathbb{C}^{n}} d \mu(\zeta) e^{-|\zeta|^{2} / 2}(\psi(\zeta))^{*}\langle z \mid \zeta, \alpha\rangle\right)^{*}= \\
& =\pi^{-n} e^{|\xi|^{2} / 2} \int_{\mathbb{C}^{n}} d \mu(\zeta) e^{-|\zeta|^{2} / 2} \psi(\zeta) \times \\
& \times \sum_{|\alpha|=m} \int_{\mathbb{C}^{n}} d \mu(z) e^{-|z|^{2}}\langle z \mid \xi, \alpha\rangle\langle z \mid \zeta, \alpha\rangle^{*}= \\
& =\pi^{-n} \int_{\mathbb{C}^{n}} d \mu(\zeta) e^{-|\zeta|^{2} / 2} \psi(\zeta) \times \\
& \times \sum_{|\alpha|=m} \frac{1}{\pi^{n} \alpha !} \int_{\mathbb{C}^{n}} d \mu(z) e^{-|z|^{2}} e^{z \cdot \xi^{*}-|\xi|^{2} / 2}\left(z-\xi^{*}\right)^{\alpha} \times \\
& \times e^{z^{*} \cdot \zeta-|\zeta|^{2} / 2}\left(z^{*}-\zeta\right)^{\alpha}= \\
& =\pi^{-n} \int_{\mathbb{C}^{n}} d \mu(\zeta) e^{-|\zeta|^{2}} \psi(\zeta) \sum_{|\alpha|=m} \frac{1}{\pi^{n} \alpha !} \int_{\mathbb{C}^{n}} \prod_{j=1}^{n} d \mu\left(z_{j}\right) e^{-\left|z_{j}\right|^{2} \times} \\
& \times e^{z_{j} \xi_{j}+z_{j}^{*} \zeta_{j}^{*}}\left(z_{j}-\xi_{j}^{*}\right)^{\alpha_{j}}\left(z_{j}^{*} \zeta_{j}\right)^{\alpha_{j}}= \\
& =\pi^{-n} \int_{\mathbb{C}^{n}} d \mu(\zeta) e^{-|\zeta|^{2}} \psi(\zeta) \sum_{|\alpha|=m} \frac{1}{\pi^{n} \alpha !} \int_{\mathbb{C}^{n}} \prod_{j=1}^{n} e^{\zeta_{j}^{*} \xi_{j}} \mathcal{I}_{j},
\end{aligned}
$$

где

$$
\begin{aligned}
\mathcal{I}_{j} & :=\int_{\mathbb{C}^{n}} d \mu\left(z_{j}\right) e^{\left|z_{j}\right|^{2}}\left(z_{j}^{*}\right)^{\alpha_{j}} \sum_{k=0}^{\alpha_{j}} \frac{\alpha_{j}}{k !\left(\alpha_{j}-k\right) !}\left(\zeta_{j}^{*}-\xi_{j}^{*}\right)^{k} z_{j}^{\alpha_{j}-k} \sum_{l=0}^{+\infty} \frac{1}{l !}\left(\zeta_{j}-\xi_{j}\right)^{l}\left(z_{j}\right)^{l}= \\
& =\sum_{\substack{0 \leqslant k \leqslant \alpha_{j} \\
0 \leqslant l<+\infty}} \frac{\alpha_{j}}{k ! l !\left(\alpha_{j}-k\right) !}\left(\int_{\mathbb{C}^{n}} d \mu\left(z_{j}\right) e^{-\left|z_{j}\right|^{2}} z_{j}^{\alpha_{j}+l}\left(z_{j}^{*}\right)^{\alpha_{j}-k}\right)\left(\xi_{j}^{*}-\zeta_{j}^{*}\right)^{k}\left(\zeta_{j}-\xi_{j}\right)^{l} .
\end{aligned}
$$

Для того чтобы вычислить интеграл в последней сумме, используем полярные координаты $z_{j}=r_{j} e^{i \theta_{j}}, r_{j}>0,0 \leqslant \theta_{j} \leqslant 2 \pi$. Получаем

$$
\begin{aligned}
\mathcal{I}_{j}= & \sum_{\substack{0 \leqslant k \leqslant \alpha_{j} \\
0 \leqslant l<+\infty}} \frac{\alpha_{j} !}{k ! l !\left(\alpha_{j}-k\right) !}\left(\xi_{j}^{*}-\zeta_{j}^{*}\right)^{k}\left(\zeta_{j}-\xi_{j}\right)^{l} \times \\
& \times \int_{0}^{2 \pi} d \theta_{j} e^{i(k-l) \theta_{j}} \int_{0}^{+\infty} d r_{j} r_{j}^{2 \alpha_{j}+1-k+l} e^{-r_{j}^{2}}
\end{aligned}
$$


После вычисления сумма $\mathcal{I}_{j}$ сводится к выражению

$$
\mathcal{I}_{j}=2 \pi \sum_{0 \leqslant k \leqslant \alpha_{j}} \frac{\alpha_{j} !}{k ! l !\left(\alpha_{j}-k\right) !} \frac{\alpha_{j}}{2}\left(\xi_{j}^{*}-\zeta_{j}^{*}\right)^{k}\left(\zeta_{j}-\xi_{j}\right)^{l} .
$$

Вспомнив, что полином Лагерра дается формулой [10]

$$
L_{p}^{\nu}(x)=\sum_{k=0}^{k=p}(-1)^{k}\left(\begin{array}{c}
p+\nu \\
p-k
\end{array}\right) \frac{x^{k}}{k !}
$$

получим

$$
\mathcal{I}_{j}=\pi \alpha_{j} ! L_{\alpha_{j}}^{0}\left(\left|\xi_{j}-\zeta_{j}\right|^{2}\right)
$$

Поэтому имеем

$$
\begin{aligned}
\widetilde{\mathcal{W}}_{m}\left[\varphi_{\psi}\right](\xi)= & \pi^{-n} \int_{\mathbb{C}^{n}} d \mu(\zeta) e^{-|\zeta|^{2}} \psi(\zeta) e^{\xi \cdot \zeta} \times \\
& \times \sum_{\alpha_{1}+\cdots+\alpha_{n}=m} L_{\alpha_{1}}^{0}\left(\left|\xi_{1}-\zeta_{1}\right|^{2}\right) \ldots L_{\alpha_{n}}^{0}\left(\left|\xi_{n}-\zeta_{n}\right|^{2}\right) .
\end{aligned}
$$

Используя формулу суммирования [10]

$$
\sum_{i_{1}+\cdots+i_{n}=p} L_{i_{1}}^{\beta_{1}}\left(x_{1}\right) \ldots L_{i_{n}}^{\beta_{n}}\left(x_{n}\right)=L_{p}^{\beta_{1}+\cdots+\beta_{n}+d-1}\left(x_{1}+\cdots+x_{n}\right)
$$

для $p=m, d=n, \beta_{j}=0, i_{j}=\alpha_{j}$ и $x_{j}=\left|\xi_{j}-\zeta_{j}\right|^{2}, 1 \leqslant j \leqslant n$, получаем

$$
\widetilde{\mathcal{W}}_{m}\left[\varphi_{\psi}\right](\xi)=\int_{\mathbb{C}^{n}} d \mu(\zeta) e^{-|\zeta|^{2}} \psi(\zeta)\left(\pi^{-n} e^{\xi \cdot \zeta} L_{m}^{n-1}\left(|\xi-\zeta|^{2}\right)\right) .
$$

Но функция переменных $\xi, \zeta$ в последнем интеграле совпадает с воспроизводящим ядром собственного пространства $\mathcal{A}_{m}^{2}\left(\mathbb{C}^{n}\right)[9]$. Поэтому последнее равенство означает, что $\widetilde{\mathcal{W}}_{m}\left[\varphi_{\psi}\right](\xi)=\psi(\xi)$. Очевидно, что $z \rightarrow \varphi_{\psi}(z)$ есть целая функция. Более того, она удовлетворяет условию $\left\langle\varphi_{\psi}, \varphi_{\psi}\right\rangle_{\mathcal{H}} \leq+\infty$, поскольку $\widetilde{\mathcal{W}}_{m}$ есть унитарное отображение. Тем самым включение $\mathcal{A}_{m}^{2}\left(\mathbb{C}^{n}\right) \subset \widetilde{\mathcal{W}}_{m}\left[\mathcal{F}_{2}\left(\mathbb{C}^{n}\right)\right]$ доказано.

\section{7. ЗАКЛЮЧЕНИЕ}

При рассмотрении четномерного аналога заряженной частишы в постоянном однородном магнитном поле для каждого уровня Ландау мы построили семейство ОКС путем сдвига фоковских состояний посредством операторов представления Фока-Баргмана группы Гейзенберга. При построении был использован метод, в основе которого лежат квадратично интегрируемые на однородном пространстве представления основной группы. Мы установили, что образ пространства Фока при преобразованиях КС, ассоциированных с построенными ОКС, совпадает с пространствами связанных состояний частицы. Это дает новое описание многомерных евклидовых состояний Ландау. Таким образом, метод КС дает нам возможность посмотреть на собственные пространства гамильтониана Ландау с математической точки зрения.

Благодарности. Автор благодарен своим коллегам Дж. Кубали, О. Мадихи и И. Такату. 


\section{Список литературы}

[1] V. V. Dodonov. J. Opt. B. Quant. Semiclass. Opt. 2002. V. 4. P. R1.

[2] И. А. Малкин, В. И. Манько. ЖЭТФ. 1969. Т. 55. С. 1014.

[3] B. R. Johnson, J. O. Hirschfelder, K. H. Yang. Rev. Mod. Phys. 1983. V. 55. P. 109.

[4] S. Varro. J. Phys. A. 1984. V. 17. P. 1631.

[5] S. M. Girvin, T. Jach. Phys. Rev. B. 1984. V. 29. P. 5617.

[6] M. K. Fung, Y. F. Wang. Chinese J. Phys. 1999. V. 37. № 5. P. 435.

[7] G. B. Folland. Proc. Amer. Math. Soc. 1975. V. 47. P. 401.

[8] W. Rudin. Function Theory in the Unit Ball of $\mathbb{C}^{n}$. New York: Springer, 1980.

[9] N. Askour, A. Intissar, Z. Mouayn. J. Math. Phys. 2000. V. 41. P. 3057.

[10] И. С. Градитейн, И. М. Рыжик. Таблицы интегралов, сумм, рядов и произведений. М.: Физматгиз, 1963.

[11] V. V. Kisil. Acta Appl. Math. 1999. V. 59. P. 79.

[12] G. B. Folland. Harmonic Analysis on Phase Space. Ann. Math. Stud. V. 122. Princeton, NJ: Princeton Univ. Press, 1989.

[13] C. Benson, J. Jenkins, G. Ratclif. J. Funct. Ann. 1992. V. 105. P. 405.

[14] M. Duflo, C. C. Moore. J. Funct. Anal. 1976. V. 21. № 2. P. 208.

Поступила в редакцию 10.VII.2003 г. 